\title{
Chers lecteurs
}

\section{S. Perrot (Rédacteur en chef)}

(C) Lavoisier SAS 2018

\section{Un nouveau journal, une nouvelle équipe éditoriale pour «Douleur et Analgésie »}

C'est un grand plaisir et une grande fierté que de présenter ce nouveau numéro de Douleur et Analgésie. Avec ce numéro, démarre une nouvelle période de ce journal qui, je l'espère, sera féconde et enthousiasmante comme l'ont été les précédentes. Merci à François Cesselin, précédent rédacteur en chef, de nous confier une si belle revue et un défi important. Autour de la SFETD, c'est une équipe éditoriale francophone renouvelée : Catherine Ferland pour le Canada, Marie Besson pour la Suisse, André Mouraux pour la Belgique, que nous vous présentons. Une nouvelle équipe pour une revue toujours plus dynamique, avec de nombreuses rubriques pour tous les professionnels de la médecine de la douleur.

\section{La douleur en santé mentale : un sujet majeur}

Ce numéro aborde la douleur et la santé mentale, sujet souvent délaissé et peu traité. Il aborde l'importance de reconnaître la douleur chez les personnes atteintes de troubles du spectre autistique, la notion de douleur psychique et des liens avec le suicide, et les différences de sexe dans la douleur.

\section{Des rubriques sur tous les sujets, pour tous}

La revue change, évolue, avec des directeurs de rubrique, pour représenter la diversité des professionnels de la douleur. Ainsi, ce numéro aborde aussi la complexité de la douleur, le monitorage de la douleur en anesthésie, la fibromyalgie pédiatrique et les douleurs postchirurgicales.

Douleur et Analgésie, c'est la revue de tous les professionnels de la douleur, c'est aussi la revue de toute la francophonie. À vous de nous lire, à vous de nous dire et aussi à vous de nous écrire! 